\title{
Kinematic analysis as the basis for training strategy in gymnastics
}

\author{
Denis Semenov ${ }^{1 *}$, Vyacheslav Shlyakhtov ${ }^{1}$, and Alexandr Rumyantsev ${ }^{1}$ \\ ${ }^{1}$ Theory and Methodology of Gymnastics Department, Velikiye Luki State Academy of Physical \\ Education and Sports, Velikiye Luki, Russia
}

\begin{abstract}
The kinematic analysis of gymnastic skills performance is a widely used research method in sports science. This study presents the experience of applying the method of kinematic analysis in the managing the training process of junior gymnasts on the example of mastering three basic gymnastic skills. The test group included 10 junior male gymnasts of the age 9 to 12. The subject of kinematic analysis was based on comparing gymnasts' joint angles, angular velocity and angular acceleration parameters. The kinematics parameters of gymnastic skills performance by a highly qualified gymnast became the target model for junior gymnasts. As a result of these targets application in the training of junior gymnasts, changes in the basic skills kinematic parameters were detected. At the end of the study, the kinematics parameters of junior gymnasts became much closer to the model parameters. Thus, the effectiveness of the kinematics analysis method in the training of junior gymnasts was demonstrated.
\end{abstract}

\section{Introduction}

Artistic gymnastics is one of the most difficult sports associated with high motor coordination of the athletes' movements. Training in gymnastics requires gymnasts to master a huge number of different exercises. The specifics of this sport, and, in particular, the peculiarities of judging gymnastic performances at competitions, involve evaluating the quality of gymnastic exercises in comparison with the model technique parameters of these exercises.

The priority tasks of training in gymnastics involve the formation of gymnasts' ability to reliably and accurately perform the competitive program skills at a high quality level. The quality of the skills is most determined by the external parameters of the movements or their kinematic characteristics. In this regard, the analysis of the kinematic parameters of gymnastic skills is a widespread and effective method of evaluating their performance effectiveness [1-8]. This method is successfully used in solving various research tasks, and is also gradually being introduced into the system of gymnastics competitions judging [9, $10]$.

\footnotetext{
* Corresponding author: semenov-den@yandex.ru
} 
Numerous scientific studies show the success of the kinematic analysis method application in solving various gymnasts training tasks $[2,3,4,16,18]$.

A number of studies $[11,12,15,16]$ have shown the use of the kinematic analysis of gymnastic skills performance in order to determine the structural relationship between the same type technique elements. It proved the possibility of a positive transfer of motor skills and allowed to offer the sequence of training in gymnastic exercises.

\section{Materials and methods}

The strategy of gymnasts training was developed on the base of basic gymnastic skills kinematic analysis. It involved the evaluating of gymnastic skills kinematic parameters performed by junior gymnasts and comparing these parameters with the model ones obtained from an experienced gymnast performance. The analysis of kinematics included the joint angles kinematics when parameters of knee joint, coxofemoral (hip) joint, humeral joint and ulnar joint from one side of the gymnast's body were examined. For these joints angular velocity and angular acceleration data were also explored.

\subsection{Content of the experiment}

To conduct the study, a group of 10 junior male gymnasts aged 9 to 12 year old was formed $(n=10)$. The kinematic parameters of three basic gymnastic skills performance by each of gymnasts were registered at the beginning of the study. A high frequency video (300 fps) and computer processing in the software package "Star Trace" were used. As a result the parameters of joint angles, angular velocities and angular accelerations dynamics of each gymnast were obtained. In the same way, the kinematic parameters of a high qualified gymnast performance of the same basic skills were registered. These parameters became models for junior gymnasts. The test group training strategy was built on the base of this target model kinematics of the basic skills performance. The experimental training period lasted for 9 months.

\subsection{Statistical analysis}

The statistical processing, analysis and systematization of the obtained data was carried out using the software Microsoft Excel and Stat Soft Statistica 10. For mathematical data processing the average values of joint angles, angular velocities, and angular accelerations were determined. To determine the reliability of the differences, the T-Student criterion was calculated. The $5 \%$ p-value was the level of statistically significant differences in kinematic parameters.

\section{Results and Discussion}

To get the objective guidelines for the managing of junior gymnasts training the kinematic parameters of the following basic skills performance by an experienced gymnast were obtained: acrobatic forward handspring, backward handspring, forward handspring vault. These kinematic parameters (joint angles, angular velocities, and angular accelerations) became models for junior gymnasts.

The comparing of the studied kinematic parameters of the test group gymnasts forward handspring performance with the model ones, significant differences were revealed in a number of parameters (Table 1). The phase of the main actions - pushing-off with the hands was studied. 
Table 1. Handspring forward joint angles kinematics.

\begin{tabular}{|l|l|l|l|l|l|}
\hline $\begin{array}{l}\text { Kinematic } \\
\text { parameters }\end{array}$ & Gymnast & Knee joint & $\begin{array}{l}\text { coxofemoral } \\
\text { (hip) joint }\end{array}$ & $\begin{array}{l}\text { humeral } \\
\text { joint }\end{array}$ & ulnar joint \\
\hline $\begin{array}{l}\text { Joint angle, } \\
\text { degree }\end{array}$ & $\begin{array}{l}\text { Highly } \\
\text { qualified }\end{array}$ & $168.1^{\circ} \pm 2.7^{\circ}$ & $160.1^{\circ} \pm 17.3^{\circ}$ & $193.7^{\circ} \pm 6.7^{\circ}$ & $179.1^{\circ} \pm 3.7^{\circ}$ \\
\cline { 2 - 6 } & Junior & $152.8^{\circ} \pm 4.8^{\circ}$ & $181.3^{\circ} \pm 14.5^{\circ}$ & $177.9^{\circ} \pm 7.4^{\circ}$ & $177.7^{\circ} \pm 1.8^{\circ}$ \\
\hline T-test & $\mathbf{p}<\mathbf{0 . 0 5}$ & $\mathrm{p}>0.05$ & $\mathbf{p}<\mathbf{0 . 0 5}$ & $\mathrm{p}>0.05$ \\
\hline $\begin{array}{l}\text { Angular } \\
\text { velocity, } \\
\text { rad/s }\end{array}$ & $\begin{array}{l}\text { Highly } \\
\text { qualified }\end{array}$ & $-0.48 \pm 0.2$ & $-2.94 \pm 0.4$ & $-1.06 \pm 0.03$ & $0.15 \pm 0.4$ \\
\cline { 2 - 6 } & Junior & $-0.41 \pm 0.3$ & $-2.28 \pm 0.9$ & $-1.13 \pm 0.3$ & $-0.14 \pm 0.3$ \\
\hline T-test & $\mathrm{p}>0.05$ & $\mathrm{p}>0.05$ & $\mathrm{p}>0.05$ & $\mathrm{p}>0.05$ \\
\hline $\begin{array}{l}\text { Angular } \\
\text { acceleration, } \\
\text { rad/s }{ }^{2}\end{array}$ & $\begin{array}{l}\text { Highly } \\
\text { qualified }\end{array}$ & $-0.62 \pm 5.2$ & $4.62 \pm 4.9$ & $-0.96 \pm 6.4$ & $-2.01 \pm 5.2$ \\
\cline { 2 - 7 } Junior & $-1.26 \pm 3.1$ & $6.18 \pm 4.1$ & $1.42 \pm 2.6$ & $2.18 \pm 1.7$ \\
\hline T-test & $\mathrm{p}>0.05$ & $\mathrm{p}>0.05$ & $\mathrm{p}>0.05$ & $\mathrm{p}>0.05$ \\
\hline
\end{tabular}

As can be seen from the results presented in Table 1, the forward handspring kinematics show the difference between the average test group parameters and the model parameters shown by the highly qualified gymnast. At the same time, statistically significant differences were found in the angular kinematics of the knee and shoulder joints, which indicates weaknesses in the training of junior gymnasts. Certain recommendations were made in the training process of junior gymnasts based on the revealed features of this skill performance.

The backward handspring kinematic analysis included the study of the two supporting phases of this skill - take off from feet and push off with hands. Target model kinematic parameters obtained in our research mostly the same as in other research works $[13,14]$. The test group kinematic parameters compared to the model ones, show significant differences in a number of parameters (Table 2).

As can be seen from the results presented in Table 2, the average junior gymnasts' group parameters of the backward handspring kinematics showed significant differences in a number of parameters from the model ones shown by highly qualified gymnast. The appropriate accents in the training of young gymnasts were made based on the identified features. 
Table 2. Handspring backward joint angles kinematics.

\begin{tabular}{|c|c|c|c|c|c|}
\hline \multirow{2}{*}{$\begin{array}{l}\text { Kinematic } \\
\text { parameters }\end{array}$} & \multirow[t]{2}{*}{ Gymnast } & \multicolumn{4}{|c|}{ Take off } \\
\hline & & Knee joint & $\begin{array}{l}\text { coxofemoral } \\
\text { (hip) joint }\end{array}$ & $\begin{array}{l}\text { humeral } \\
\text { joint }\end{array}$ & ulnar joint \\
\hline \multirow[t]{2}{*}{$\begin{array}{l}\text { Joint angle, } \\
\text { degree }\end{array}$} & $\begin{array}{l}\text { Highly } \\
\text { qualified }\end{array}$ & $124.1^{\circ} \pm 4.2^{\circ}$ & $213.8^{\circ} \pm 35.1^{\circ}$ & $214.4^{\circ} \pm 9.9^{\circ}$ & $174.1^{\circ} \pm 3.2^{\circ}$ \\
\hline & Junior & $133.9^{\circ} \pm 6.5^{\circ}$ & $203.2^{\circ} \pm 25.2^{\circ}$ & $196.7^{\circ} \pm 6.6^{\circ}$ & $178.2^{\circ} \pm 6.6^{\circ}$ \\
\hline \multicolumn{2}{|l|}{ T-test } & $\mathrm{p}<0.05$ & $\mathrm{p}<\mathbf{0 . 0 5}$ & p $<0.05$ & $p>0.05$ \\
\hline \multirow[t]{2}{*}{$\begin{array}{l}\text { Angular } \\
\text { velocity, rad/s }\end{array}$} & $\begin{array}{l}\text { Highly } \\
\text { qualified }\end{array}$ & $-0.59 \pm 0.2$ & $-5.67 \pm 1.6$ & $-1.91 \pm 0.9$ & $-0.59 \pm 0.4$ \\
\hline & Junior & $-1.19 \pm 1.4$ & $-4.91 \pm 0.9$ & $-1.19 \pm 0.6$ & $-1.08 \pm 0.6$ \\
\hline \multicolumn{2}{|l|}{ T-test } & $p>0.05$ & $p>0.05$ & $p>0.05$ & $p>0.05$ \\
\hline \multirow{2}{*}{$\begin{array}{l}\text { Angular } \\
\text { acceleration, } \\
\mathrm{rad} / \mathrm{s}^{2}\end{array}$} & $\begin{array}{l}\text { Highly } \\
\text { qualified }\end{array}$ & $9.0 \pm 2.1$ & $-14.1 \pm 10.3$ & $-8.7 \pm 5.4$ & $1.8 \pm 1.2$ \\
\hline & Junior & $15.8 \pm 4.0$ & $5.5 \pm 17.4$ & $-6.5 \pm 7.5$ & $5.8 \pm 3.1$ \\
\hline \multicolumn{2}{|l|}{ T-test } & $\mathrm{p}<0.05$ & $p<0.05$ & $\mathrm{p}<0.05$ & $\mathrm{p}<0.05$ \\
\hline & & \multicolumn{4}{|c|}{ Push off } \\
\hline \multirow[t]{2}{*}{$\begin{array}{ll}\text { Joint } & \text { angle, } \\
\text { degree } & \end{array}$} & $\begin{array}{l}\text { Highly } \\
\text { qualified }\end{array}$ & $148.9^{\circ} \pm 11.8^{\circ}$ & $171.4^{\circ} \pm 26.3^{\circ}$ & $205.5^{\circ} \pm 14.5^{\circ}$ & $176.3^{\circ} \pm 2.8^{\circ}$ \\
\hline & Junior & $147.6^{\circ} \pm 5.0^{\circ}$ & $189.7^{\circ} \pm 17.5^{\circ}$ & $192.8^{\circ} \pm 6.1^{\circ}$ & $173.9^{\circ} \pm 3.3^{\circ}$ \\
\hline \multicolumn{2}{|l|}{ T-test } & $p>0.05$ & $\mathbf{p}<\mathbf{0 . 0 5}$ & $\mathbf{p}<\mathbf{0 . 0 5}$ & $p>0.05$ \\
\hline \multirow[t]{2}{*}{$\begin{array}{l}\text { Angular } \\
\text { velocity, } \mathrm{rad} / \mathrm{s}\end{array}$} & $\begin{array}{l}\text { Highly } \\
\text { qualified }\end{array}$ & $1.64 \pm 0.6$ & $4.35 \pm 1.6$ & $2.07 \pm 0.5$ & $0.49 \pm 0.5$ \\
\hline & Junior & $0.9 \pm 0.6$ & $3.26 \pm 0.7$ & $1.23 \pm 0.3$ & $-0.42 \pm 0.4$ \\
\hline \multicolumn{2}{|l|}{ T-test } & $p>0.05$ & $\mathrm{p}<\mathbf{0 . 0 5}$ & $p>0.05$ & $p>0.05$ \\
\hline \multirow{2}{*}{$\begin{array}{l}\text { Angular } \\
\text { acceleration, } \\
\mathrm{rad} / \mathrm{s}^{2}\end{array}$} & $\begin{array}{l}\text { Highly } \\
\text { qualified }\end{array}$ & $3.3 \pm 1.4$ & $4.2 \pm 12.7$ & $-5.9 \pm 8.7$ & $-2.8 \pm 2.9$ \\
\hline & Junior & $-7.6 \pm 6.4$ & $8.4 \pm 19.4$ & $5.1 \pm 7.3$ & $1.0 \pm 3.1$ \\
\hline \multicolumn{2}{|l|}{ T-test } & $\mathbf{p}<\mathbf{0 . 0 5}$ & $\mathrm{p}<0.05$ & $\mathbf{p}<\mathbf{0 . 0 5}$ & $\mathrm{p}<0.05$ \\
\hline
\end{tabular}

The same way the forward handspring vault kinematics was analyzed (Table 3 ). The same - take off and push off phases were studied in this skill. There are many research works done where specifics of different vaults technique efficiency described. The data presented in our research match to main results of these works $[15,17,18]$.

The results presented in Table 3 also indicate differences in the kinematic parameters of the forward handspring vault performed by junior gymnasts and the high qualified gymnast. Statistically significant differences were found in the kinematics of the knee, elbow, and hip angles. Because the interaction of individual units of biokinematic chain is critical in gymnastic skills support phases execution, it is important for gymnast to reproduce the motor action with the most efficient angular parameters in the joints of the body to achieve maximum result in push off action performance. The revealed differences in the kinematics of this skill performance determined the guidelines for managing the training process of junior gymnasts. 
Table 3. Handspring forward vault joint angles kinematics.

\begin{tabular}{|c|c|c|c|c|c|}
\hline \multirow{2}{*}{$\begin{array}{l}\text { Kinematic } \\
\text { parameters }\end{array}$} & \multirow[t]{2}{*}{ Gymnast } & \multicolumn{4}{|c|}{ Take off } \\
\hline & & Knee joint & $\begin{array}{l}\text { coxofemoral } \\
\text { (hip) joint }\end{array}$ & humeral joint & ulnar joint \\
\hline \multirow[t]{2}{*}{$\begin{array}{l}\text { Joint angle, } \\
\text { degree }\end{array}$} & $\begin{array}{l}\text { Highly } \\
\text { qualified }\end{array}$ & $196.6^{\circ} \pm 1.1^{\circ}$ & $143.7^{\circ} \pm 2.5^{\circ}$ & $112.5^{\circ} \pm 23.2^{\circ}$ & $191.2^{\circ} \pm 6.6^{\circ}$ \\
\hline & Junior & $190.3^{\circ} \pm 9.7^{\circ}$ & $140.1^{\circ} \pm 9.1^{\circ}$ & $122.5^{\circ} \pm 35.6^{\circ}$ & $206.4^{\circ} \pm 13.3^{\circ}$ \\
\hline \multicolumn{2}{|l|}{ T-test } & $p>0.05$ & $p>0.05$ & $p>0.05$ & $\mathrm{p}<\mathbf{0 . 0 5}$ \\
\hline \multirow[t]{2}{*}{$\begin{array}{l}\text { Angular } \\
\text { velocity, } \mathrm{rad} / \mathrm{s}\end{array}$} & $\begin{array}{l}\text { Highly } \\
\text { qualified }\end{array}$ & $-0.16 \pm 0.3$ & $0.37 \pm 1.2$ & $-1.31 \pm 5.5$ & $-2.36 \pm 1.3$ \\
\hline & Junior & $3.8 \pm 2.0$ & $-2.1 \pm 2.0$ & $0.21 \pm 3.5$ & $-2.9 \pm 1.6$ \\
\hline \multicolumn{2}{|l|}{ T-test } & $\mathbf{p}<0.05$ & $p>0.05$ & $p>0.05$ & $p>0.05$ \\
\hline \multirow{2}{*}{$\begin{array}{l}\text { Angular } \\
\text { acceleration, } \\
\mathrm{rad} / \mathrm{s}^{2}\end{array}$} & $\begin{array}{l}\text { Highly } \\
\text { qualified }\end{array}$ & $-5.4 \pm 4.3$ & $23.1 \pm 4.9$ & $61.3 \pm 37.4$ & $17.6 \pm 5.5$ \\
\hline & Junior & $-23.7 \pm 10.4$ & $19.8 \pm 6.2$ & $18.3 \pm 9.7$ & $19.3 \pm 8.2$ \\
\hline \multicolumn{2}{|l|}{ T-test } & $p>0.05$ & $p>0.05$ & $p>0.05$ & $p>0.05$ \\
\hline & & \multicolumn{4}{|l|}{ Push off } \\
\hline \multirow[t]{2}{*}{$\begin{array}{l}\text { Joint angle, } \\
\text { degree }\end{array}$} & $\begin{array}{l}\text { Highly } \\
\text { qualified }\end{array}$ & $187.9^{\circ} \pm 3.5^{\circ}$ & $190.0^{\circ} \pm 1.3^{\circ}$ & $157.1^{\circ} \pm 4.8^{\circ}$ & $183.3^{\circ} \pm 2.0^{\circ}$ \\
\hline & Junior & $225.4^{\circ} \pm 6.5^{\circ}$ & $184.1^{\circ} \pm 21.6^{\circ}$ & $150.0^{\circ} \pm 14.9^{\circ}$ & $199.2^{\circ} \pm 2.3^{\circ}$ \\
\hline \multicolumn{2}{|l|}{ T-test } & $\mathbf{p}<\mathbf{0 . 0 5}$ & $p>0.05$ & $p>0.05$ & p $<0.05$ \\
\hline \multirow[t]{2}{*}{$\begin{array}{l}\text { Angular } \\
\text { velocity, } \mathrm{rad} / \mathrm{s}\end{array}$} & $\begin{array}{l}\text { Highly } \\
\text { qualified }\end{array}$ & $-0.79 \pm 0.1$ & $0.21 \pm 0.7$ & $1.08 \pm 0.4$ & $0.21 \pm 0.1$ \\
\hline & Junior & $0.01 \pm 1.4$ & $2.05 \pm 1.5$ & $1.52 \pm 1.5$ & $-0.11 \pm 1.1$ \\
\hline \multicolumn{2}{|l|}{ T-test } & $\mathbf{p}<\mathbf{0 . 0 5}$ & $\mathbf{p}<\mathbf{0 . 0 5}$ & $p>0.05$ & $p>0.05$ \\
\hline \multirow{2}{*}{$\begin{array}{l}\text { Angular } \\
\text { acceleration, } \\
\mathrm{rad} / \mathrm{s}^{2}\end{array}$} & $\begin{array}{l}\text { Highly } \\
\text { qualified }\end{array}$ & $-1.1 \pm 2.1$ & $-8.8 \pm 7.5$ & $-4.8 \pm 3.9$ & $1.0 \pm 2.1$ \\
\hline & Junior & $-5.7 \pm 6.4$ & $-4.6 \pm 9.4$ & $3.2 \pm 10.7$ & $-0.3 \pm 5.6$ \\
\hline \multicolumn{2}{|l|}{ T-test } & $\mathrm{p}>0.05$ & $p>0.05$ & $\mathbf{p}<\mathbf{0 . 0 5}$ & $p>0.05$ \\
\hline
\end{tabular}

An example of differences in joint angles kinematics is presented in figure 1. As we can see, the highly qualified gymnast joint angles kinematics graphs are smoother comparing to junior gymnast's kinematics graphs.

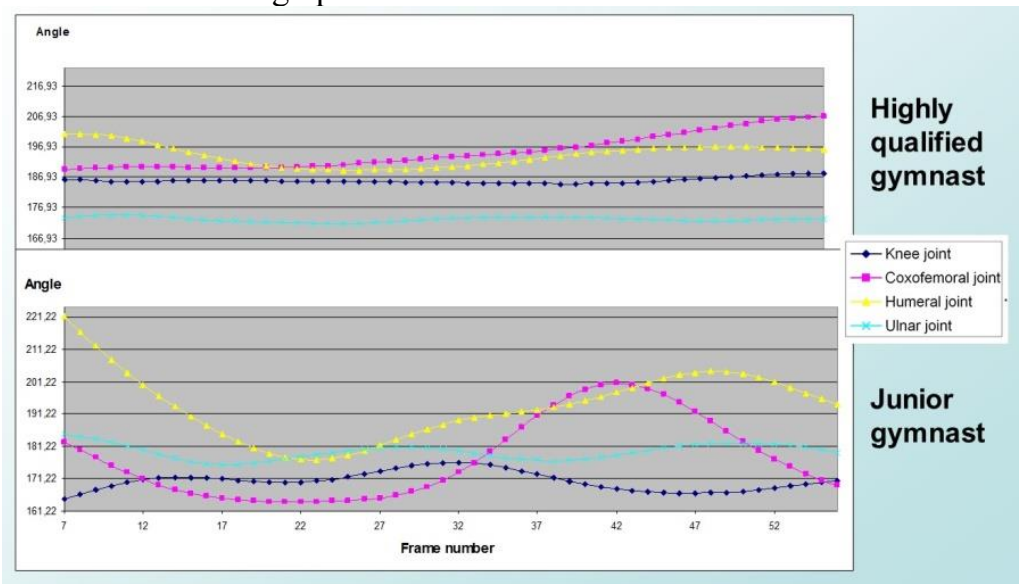

Fig. 1. Comparing of joint angles kinematics of highly qualified and junior gymnasts.

A repeated study of the basic gymnastic skills kinematics was carried out at the end of research. All junior gymnasts were assessed in performance of the same gymnastic skills. 
The data obtained were compared with the results of a preliminary study and with the target kinematic parameters of these skills. The forward handspring kinematics indicates the presence of dynamics of indicators at the end of the experiment (Table 4). Junior gymnasts began to perform the leg swing action with a more straightened leg, the value of the angle in the hip joint became closer to the value of highly qualified gymnasts, and that is, the leg swing became much more powerful. The value of the humeral joint angle has become almost the same as the target one (high qualified gymnast parameter), which indicates the locking nature of the repulsion (push off action). At the same time, the gymnasts' arms began to bend slightly more at the elbow joint, which is due to the increased effort and the depreciation caused by it.

Table 4. Handspring forward joint angles kinematics in repeated research.

\begin{tabular}{|c|c|c|c|c|c|}
\hline $\begin{array}{l}\text { Kinematic } \\
\text { parameters }\end{array}$ & Gymnast & Knee joint & $\begin{array}{l}\text { coxofemoral } \\
\text { (hip) joint }\end{array}$ & $\begin{array}{l}\text { humeral } \\
\text { joint }\end{array}$ & ulnar joint \\
\hline \multirow[t]{2}{*}{$\begin{array}{l}\text { Joint angle, } \\
\text { degree }\end{array}$} & $\begin{array}{l}\text { Highly } \\
\text { qualified }\end{array}$ & $168,1^{\circ} \pm 2,7^{\circ}$ & $160,1^{\circ} \pm 17,3^{\circ}$ & $193,7^{\circ} \pm 6,7^{\circ}$ & $179,1^{\circ} \pm 3,7^{\circ}$ \\
\hline & Junior & $165.0^{\circ} \pm 3,0^{\circ}$ & $162.5^{\circ} \pm 17,5^{\circ}$ & $191.4^{\circ} \pm 8,4^{\circ}$ & $175.0^{\circ} \pm 2,9^{\circ}$ \\
\hline \multicolumn{2}{|l|}{ T-test } & $p>0,05$ & $p>0,05$ & $\mathrm{p}>0,05$ & $p>0,05$ \\
\hline \multirow{2}{*}{$\begin{array}{l}\text { Angular } \\
\text { velocity, } \\
\mathrm{rad} / \mathrm{s}\end{array}$} & $\begin{array}{l}\text { Highly } \\
\text { qualified }\end{array}$ & $-0,48 \pm 0,2$ & $-2,94 \pm 0,4$ & $-1,06 \pm 0,03$ & $0,15 \pm 0,4$ \\
\hline & Junior & $-0,42 \pm 0,4$ & $-2,8 \pm 0,9$ & $-1,03 \pm 0,04$ & $0,2 \pm 0,2$ \\
\hline \multicolumn{2}{|l|}{ T-test } & $p>0,05$ & $p>0,05$ & $p>0,05$ & $p>0,05$ \\
\hline \multirow{2}{*}{$\begin{array}{l}\text { Angular } \\
\text { acceleration, } \\
\mathrm{rad} / \mathrm{s}^{2}\end{array}$} & $\begin{array}{l}\text { Highly } \\
\text { qualified }\end{array}$ & $-0,62 \pm 5,2$ & $4,62 \pm 4,9$ & $-0,96 \pm 6,4$ & $-2,01 \pm 5,2$ \\
\hline & Junior & $-1,1 \pm 5,4$ & $5,49 \pm 4,9$ & $-0,54 \pm 6,2$ & $-0,32 \pm 6,2$ \\
\hline \multicolumn{2}{|l|}{ T-test } & $p>0,05$ & $p>0,05$ & $\mathrm{p}>0,05$ & $p>0,05$ \\
\hline
\end{tabular}

As we can see on the table 4 the values of junior gymnasts' joint angles, angular velocities and angular accelerations changed in the target direction. There were not detected significant differences between the junior gymnasts and high qualified gymnast kinematic parameters.

Thus, the kinematics of forward handspring performed by junior gymnasts became closer in their values to the kinematics of highly qualified gymnast performance, which indicates the effectiveness of the exercise and the success of its development.

An example of joint angles kinematic parameters comparing while forward handspring performance by highly qualified gymnast and junior gymnasts at the beginning of research and at the end of research is presented in figure 2. We can assess the dynamics of angular kinematic parameters changes and its coming closer to highly qualified gymnast parameters. 


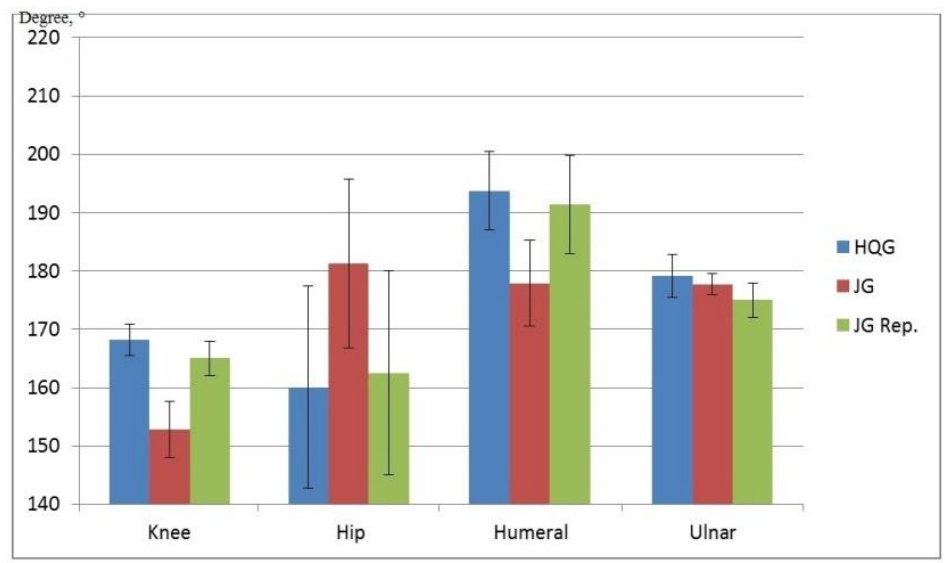

Fig. 2. Comparing of joint angles kinematic parameters while forward handspring performance by high qualified gymnast (HQG), junior gymnasts at the beginning (JG) and at the end of research (JG Rep.).

The backward handspring kinematic parameters analyzed at the end of research are also showed a noticeable dynamics of the data (Table 5).

Table 5. Backward handspring joint angles kinematics in repeated research.

\begin{tabular}{|c|c|c|c|c|c|}
\hline \multirow{2}{*}{$\begin{array}{l}\text { Kinematic } \\
\text { parameters }\end{array}$} & \multirow[t]{2}{*}{ Gymnast } & \multicolumn{4}{|c|}{ Take off } \\
\hline & & Knee joint & $\begin{array}{l}\text { coxofemoral } \\
\text { (hip) joint }\end{array}$ & humeral joint & ulnar joint \\
\hline \multirow[t]{2}{*}{$\begin{array}{l}\text { Joint angle, } \\
\text { degree }\end{array}$} & $\begin{array}{l}\text { Highly } \\
\text { qualified }\end{array}$ & $124,1^{\circ} \pm 4,2^{\circ}$ & $213,8^{\circ} \pm 35,1^{\circ}$ & $214,4^{\circ} \pm 9,9^{\circ}$ & $174,1^{\circ} \pm 3,2^{\circ}$ \\
\hline & Junior & $128,9^{\circ} \pm 4,8^{\circ}$ & $208,1^{\circ} \pm 31,0^{\circ}$ & $206,4^{\circ} \pm 19,2^{\circ}$ & $175,1^{\circ} \pm 3,6^{\circ}$ \\
\hline \multicolumn{2}{|l|}{ T-test } & $\mathrm{p}<0,05$ & $\mathrm{p}<0,05$ & $p>0,05$ & $p>0,05$ \\
\hline \multirow[t]{2}{*}{$\begin{array}{l}\text { Angular } \\
\text { velocity, } \mathrm{rad} / \mathrm{s}\end{array}$} & $\begin{array}{l}\text { Highly } \\
\text { qualified }\end{array}$ & $-0,59 \pm 0,2$ & $-5,67 \pm 1,6$ & $-1,91 \pm 0,9$ & $-0,59 \pm 0,4$ \\
\hline & Junior & $-0,9 \pm 1,4$ & $-5,3 \pm 0,8$ & $-1,74 \pm 1,5$ & $-0,53 \pm 0,6$ \\
\hline \multicolumn{2}{|l|}{ T-test } & $p>0,05$ & $p>0,05$ & $p>0,05$ & $p>0,05$ \\
\hline \multirow{2}{*}{$\begin{array}{l}\text { Angular } \\
\mathrm{acceleration,} \\
\mathrm{rad} / \mathrm{s}^{2}\end{array}$} & $\begin{array}{l}\text { Highly } \\
\text { qualified }\end{array}$ & $9,0 \pm 2,1$ & $-14,1 \pm 10,3$ & $-8,7 \pm 5,4$ & $1,8 \pm 1,2$ \\
\hline & Junior & $12,9 \pm 2,9$ & $-3,6 \pm 12,9$ & $-6,24 \pm 7,2$ & $1,4 \pm 2,2$ \\
\hline \multicolumn{2}{|l|}{ T-test } & $\mathrm{p}<0,05$ & $p<0,05$ & $\mathrm{p}<0,05$ & $\mathrm{p}<0,05$ \\
\hline & & \multicolumn{4}{|l|}{ Push off } \\
\hline \multirow[t]{2}{*}{$\begin{array}{l}\text { Joint angle, } \\
\text { degree }\end{array}$} & $\begin{array}{l}\text { Highly } \\
\text { qualified }\end{array}$ & $148,9^{\circ} \pm 11,8^{\circ}$ & $171,4^{\circ} \pm 26,3^{\circ}$ & $205,5^{\circ} \pm 14,5^{\circ}$ & $176,3^{\circ} \pm 2,8^{\circ}$ \\
\hline & Junior & $148,7^{\circ} \pm 2,4^{\circ}$ & $178,8^{\circ} \pm 3,1^{\circ}$ & $199,7^{\circ} \pm 3,1^{\circ}$ & $175,0^{\circ} \pm 0,8^{\circ}$ \\
\hline \multicolumn{2}{|l|}{ T-test } & $p>0,05$ & $p<0,05$ & $p<0,05$ & $p>0,05$ \\
\hline \multirow[t]{2}{*}{$\begin{array}{l}\text { Angular } \\
\text { velocity, } \mathrm{rad} / \mathrm{s}\end{array}$} & $\begin{array}{l}\text { Highly } \\
\text { qualified }\end{array}$ & $1,64 \pm 0,6$ & $4,35 \pm 1,6$ & $2,07 \pm 0,5$ & $0,49 \pm 0,5$ \\
\hline & Junior & $1,47 \pm 0,6$ & $3,87 \pm 1,2$ & $1,77 \pm 0,7$ & $-0,36 \pm 0,3$ \\
\hline \multicolumn{2}{|l|}{ T-test } & $\mathrm{p}>0,05$ & $\mathrm{p}>0,05$ & $\mathrm{p}>0,05$ & $\mathrm{p}>0,05$ \\
\hline \multirow{2}{*}{$\begin{array}{l}\text { Angular } \\
\text { acceleration, } \\
\mathrm{rad} / \mathrm{s}^{2}\end{array}$} & $\begin{array}{l}\text { Highly } \\
\text { qualified }\end{array}$ & $3,3 \pm 1,4$ & $4,2 \pm 12,7$ & $-5,9 \pm 8,7$ & $-2,8 \pm 2,9$ \\
\hline & Junior & $-1,3 \pm 1,6$ & $7,1 \pm 15,6$ & $-0,49 \pm 7,4$ & $-0,89 \pm 2,3$ \\
\hline \multicolumn{2}{|l|}{ T-test } & $\mathrm{p}<0,05$ & $\mathrm{p}<0,05$ & $\mathrm{p}<0,05$ & $\mathrm{p}<0,05$ \\
\hline
\end{tabular}


Most of the junior gymnasts' kinematic parameters became closer in their values to the target high qualified gymnast's kinematic parameters. That is, the junior gymnasts' technique performance became more effective, which was clearly visible visually. Despite the fact that there are still some statistically significant differences in the backward handspring kinematics between junior gymnasts and a high qualified gymnast results, it is indicated that such differences have become significantly less. This way the control of the kinematic parameters allowed to make the visual assessment of the skill performance more objective.

Similar conclusions can be drawn from the results of repeated kinematic analysis of the handspring forward vault (Table 6).

Table 6. Handspring forward vault joint angles kinematics in repeated research.

\begin{tabular}{|c|c|c|c|c|c|}
\hline \multirow{2}{*}{$\begin{array}{l}\text { Kinematic } \\
\text { parameters }\end{array}$} & \multirow[t]{2}{*}{ Gymnast } & \multicolumn{4}{|c|}{ Take off } \\
\hline & & Knee joint & $\begin{array}{l}\text { coxofemoral } \\
\text { (hip) joint }\end{array}$ & humeral joint & ulnar joint \\
\hline \multirow[t]{2}{*}{$\begin{array}{l}\text { Joint angle, } \\
\text { degree }\end{array}$} & $\begin{array}{l}\text { Highly } \\
\text { qualified }\end{array}$ & $196,6^{\circ} \pm 1,1^{\circ}$ & $143,7^{\circ} \pm 2,5^{\circ}$ & $112,5^{\circ} \pm 23,2^{\circ}$ & $191,2^{\circ} \pm 6,6^{\circ}$ \\
\hline & Junior & $195,0^{\circ} \pm 3,4^{\circ}$ & $142,8^{\circ} \pm 4,1^{\circ}$ & $115,0^{\circ} \pm 28,6^{\circ}$ & $195,0^{\circ} \pm 9,3^{\circ}$ \\
\hline \multicolumn{2}{|l|}{ T-test } & $p>0,05$ & $p>0,05$ & $p>0,05$ & $p>0,05$ \\
\hline \multirow[t]{2}{*}{$\begin{array}{l}\text { Angular } \\
\text { velocity, } \mathrm{rad} / \mathrm{s}\end{array}$} & $\begin{array}{l}\text { Highly } \\
\text { qualified }\end{array}$ & $-0,16 \pm 0,3$ & $0,37 \pm 1,2$ & $-1,31 \pm 5,5$ & $-2,36 \pm 1,3$ \\
\hline & Junior & $0,87 \pm 0,7$ & $-0,2 \pm 1,4$ & $-0,92 \pm 5,0$ & $-2,49 \pm 1,4$ \\
\hline \multicolumn{2}{|l|}{ T-test } & $p>0,05$ & $p>0,05$ & $p>0,05$ & $p>0,05$ \\
\hline \multirow{2}{*}{$\begin{array}{l}\text { Angular } \\
\text { acceleration, } \\
\mathrm{rad} / \mathrm{s}^{2}\end{array}$} & $\begin{array}{l}\text { Highly } \\
\text { qualified }\end{array}$ & $-5,4 \pm 4,3$ & $23,1 \pm 4,9$ & $61,3 \pm 37,4$ & $17,6 \pm 5,5$ \\
\hline & Junior & $-9,9 \pm 6,4$ & $22,3 \pm 5,2$ & $50,6 \pm 30,4$ & $18,0 \pm 6,3$ \\
\hline \multicolumn{2}{|l|}{ T-test } & $p>0,05$ & $p>0,05$ & $p>0,05$ & $p>0,05$ \\
\hline & & \multicolumn{4}{|l|}{ Push off } \\
\hline \multirow[t]{2}{*}{$\begin{array}{l}\text { Joint angle, } \\
\text { degree }\end{array}$} & $\begin{array}{l}\text { Highly } \\
\text { qualified }\end{array}$ & $187,9^{\circ} \pm 3,5^{\circ}$ & $190,0^{\circ} \pm 1,3^{\circ}$ & $157,1^{\circ} \pm 4,8^{\circ}$ & $183,3^{\circ} \pm 2,0^{\circ}$ \\
\hline & Junior & $197,3^{\circ} \pm 4,2^{\circ}$ & $188,5^{\circ} \pm 6,4^{\circ}$ & $155,3^{\circ} \pm 7,3^{\circ}$ & $187,3^{\circ} \pm 2,0^{\circ}$ \\
\hline \multicolumn{2}{|l|}{ T-test } & $p<0,05$ & $p>0,05$ & $p>0,05$ & $\mathrm{p}<0,05$ \\
\hline \multirow[t]{2}{*}{$\begin{array}{l}\text { Angular } \\
\text { velocity, rad/s }\end{array}$} & $\begin{array}{l}\text { Highly } \\
\text { qualified }\end{array}$ & $-0,79 \pm 0,1$ & $0,21 \pm 0,7$ & $1,08 \pm 0,4$ & $0,21 \pm 0,1$ \\
\hline & Junior & $-0,5 \pm 0,4$ & $0,67 \pm 1,0$ & $1,18 \pm 0,7$ & $0,18 \pm 0,4$ \\
\hline \multicolumn{2}{|l|}{ T-test } & $p>0,05$ & $p<0,05$ & $p>0,05$ & $p>0,05$ \\
\hline \multirow{2}{*}{$\begin{array}{l}\text { Angular } \\
\text { acceleration, } \\
\mathrm{rad} / \mathrm{s}^{2}\end{array}$} & $\begin{array}{l}\text { Highly } \\
\text { qualified }\end{array}$ & $-1,1 \pm 2,1$ & $-8,8 \pm 7,5$ & $-4,8 \pm 3,9$ & $1,0 \pm 2,1$ \\
\hline & Junior & $-2,3 \pm 3,6$ & $-6,3 \pm 7,1$ & $-2,8 \pm 5,6$ & $0,7 \pm 3,7$ \\
\hline \multicolumn{2}{|l|}{ T-test } & $p>0,05$ & $p>0,05$ & $p>0,05$ & $p>0,05$ \\
\hline
\end{tabular}

The changes in dynamics of joint angles kinematics have a direct tendency to the model parameters established from a high qualified gymnast performance. According to the values of angular velocities and angular accelerations, there was also a noticeable dynamics of the indicators in the direction of the model parameters. Statistically significant differences between the kinematic parameters of junior gymnasts and highly qualified gymnast have become less, and in general, the junior gymnasts' technique of handspring forward vault performance has become more rational. Thus, the handspring forward vault kinematic parameters of junior gymnasts became in their values closer to the kinematic parameters of the highly qualified gymnast that demonstrates the effectiveness of the exercise and the success of its development. 


\section{Conclusions}

Based on the conducted research and the results obtained during the experiment, it is possible to draw convincing conclusions about the effectiveness and expediency of using the method of kinematic analysis for the gymnastic skills technique performance evaluating. In turn, the obtained kinematic parameters become effective tools for an objective assessment of the quality of the exercise, and also characterize directions of the gymnast's individual movements and actions technique performance correction. These facts allow to make specific instructions in the gymnasts training process on the basis of objective data. Hence, it is important to use the kinematic analysis method in the educational and training process both in gymnastics and other sports.

\section{References}

1. S. Prassas, Y-H. Kwon, WA. Sands, Biomechanical research in artistic gymnastics: a review, Sports Biomech., 5: 261-91 (2006)

2. M. Błażkiewicz, A. Kępczyński, A. Wit, Comparative analysis of kinetics parameters during different landing after split front leaps, Pol. J. Sport Tourism, 26(2), 3-6 (2019)

3. G. Dallas, A.S. Theodorou, The influence of a hurdle target point on the kinematics of the handspring vault approach run during training, Sports Biomechanics, 19, 467-482 (2020)

4. D.S. Cerasela, D. Andrei, M.S. Nicoleta, D.M. Petru, B.I. Silviu, N. Adrian, A. Veronica, Kinematic quantification of knee joint asymmetry during preparatory phase of a standing backward tucked salto, In Proceedings of 18th International Symposium on Intelligent Systems and Informatics, SISY 2020; Subotica; Serbia; September 1719 2020, 49-54 (2020)

5. S. Coppola, D. Albano, I. Sivoccia, R. Vastola, Biomechanical analysis of a rhythmic gymnastics jump performed using two run-up techniques, Journal of Physical Education and Sport, Vol.20 (1), 37 - 42 (2020)

6. V.A. Potop, Rafal Grad, O.A. Omelyanchik, Marta Begajlo, V.N. Boloban, Element nodes of sports equipment double back flip factions and double back flip hunched performed gymnast in floor exercise, Pedagogics, psychology, medical-biological problems of physical training and sports, 7, 23-30 (2014)

7. S.M.B. Fernandes, P. Carrara, J.C. Serrão, A.C. Amadio, L. Mochizuki, Kinematic variables of table vault on artistic gymnastics, Rev. Bras. Educ. Fís. Esporte, (São Paulo), 30(1), 97-107 (2016)

8. B. Mkaouer, M. Jemni, S. Amara, H. Chaabène, Z. Tabka, Kinematic and kinetic analysis of two gymnastics acrobatic series to performing the backward stretched somersault, Journal of Human Kinetics, 37, 17-26 (2013)

9. M. Mack, Exploring cognitive and perceptual judgment processes in gymnastics using essential kinematics information, Advances in Cognitive Psychology, 16, Issue 1, 3444 (2020)

10. J. Omorczyk, L. Nosiadek, T. Ambroży, A. Nosiadek, High-frequency video capture and a computer program with frame-by-frame angle determination functionality as tools that support judging in artistic gymnastics, Acta of Bioengineering \& Biomechanics, 17(3), 85-93 (2015)

11. D.V. Semenov, V.N. Shlyakhtov, A.A. Rumyantsev, Strength display during gymnastic exercises, Teoriya i Praktika Fizicheskoy Kultury, 6, 55-58 (2015) 
12. D.V. Semenov, V.N. Shlyakhtov, A.A. Rumyantsev, Kinematic analysis of «underswing» action on high bar and parallel bars as method of skills training progression establishment, Proceedings of 8th International Scientific Conference on Kinesiology; Opatija, Croatia, May 10 - 14, 2017,175 (2017)

13. J. Omorczyk, L. Nosiadek, R. Staszkiewicz, P. Bujas, E. Puszczałowska-Lizis, Comparison of back handspring technique performed by advanced artistic gymnasts a case study, Pol. J. Sport Tourism, 24, 86-90 (2017)

14. R. Farana, D. Jandacka, G. Irwin, Influence of different hand positions on impact forces and elbow loading during the round off in gymnastics: A case study, Science of Gymnastics Journal 5(2), 5-9 (2013)

15. A. Kochanowicz, K. Kochanowicz, B. Niespodziński, J. Mieszkowski, P. Aschenbrenner, G. Bielec, M. Szark-Eckardt, Maximal power of the lower limbs of youth gymnasts and biomechanical indicators of the forward handspring vault versus the sports result, Journal of Human Kinetics, 53, 33-40 (2016)

16. K. Spencer, M. Schuhmann, The Influence of Body Position on the Straddled Tkatchev's Flight Phase in Men's Horizontal Bar. Journal of Human Sport and Exercise, 12(1), 204-218 (2017)

17. A. Atiković, New Regression Models to Evaluate the Relationship between Biomechanics of Gymnastic Vault and Initial Vault Difficulty Values. J Hum Kinet, 35: 119-126 (2012)

18. MJ Hiley, MI Jackson, MR Yeadon, Optimal technique for maximal forward rotating vaults in men's gymnastics. Hum Mov Sci., 42: 117-31 (2015). 